\title{
Cosmic rays as regulators of molecular cloud properties
}

\author{
M. Padovani ${ }^{1,2, *}$, P. Hennebelle ${ }^{3}$, and D. Galli ${ }^{2}$ \\ ${ }^{1}$ Laboratoire de Radioastronomie Millimétrique, UMR 8112 du CNRS, École Normale Supérieure et \\ Observatoire de Paris, 24 rue Lhomond, 75231 Paris cedex 05, France \\ ${ }^{2}$ INAF-Osservatorio Astrofisico di Arcetri, Largo E. Fermi 5, 50125 Firenze, Italy \\ ${ }^{3}$ CEA, IRFU, SAp, Centre de Saclay, 91191 Gif-Sur-Yvette, France \\ *now at: Laboratoire Univers et Particules de Montpellier, UMR 5299 du CNRS, Université de Montpellier 2, \\ place E. Bataillon, cc072, 34095 Montpellier, France
}

Correspondence to: M. Padovani (marco.padovani@lupm.univ-montp2.fr)

Received: 27 February 2014 - Revised: 3 June 2014 - Accepted: 5 June 2014 - Published: 27 June 2014

\begin{abstract}
Cosmic rays are the main agents in controlling the chemical evolution and setting the ambipolar diffusion time of a molecular cloud. We summarise the processes causing the energy degradation of cosmic rays due to their interaction with molecular hydrogen, focusing on the magnetic effects that influence their propagation. Making use of magnetic field configurations generated by numerical simulations, we show that the increase of the field line density in the collapse region results in a reduction of the cosmic-ray ionisation rate. As a consequence the ionisation fraction decreases, facilitating the decoupling between the gas and the magnetic field.
\end{abstract}

\section{Introduction}

Low-mass prestellar cores are the basic units of star formation in nearby clouds like Taurus and Perseus, where stars like our Sun have been forming over the last few million years. The study of the physical structure and kinematics of these cores is therefore crucial for our understanding of the star formation process. Moreover, prestellar cores are ideal laboratories for interstellar medium chemistry which can be modelled using observations with different tracers.

Cosmic rays (hereafter CRs) have a leading role in the dynamics and chemistry of the interstellar medium (ISM). The energy density of CRs with energies $E \gtrsim 1 \mathrm{GeV}$ is about $1 \mathrm{eV} \mathrm{cm}^{-3}$. This value is comparable to that present in the Galactic magnetic field, to the energy density of the cosmic microwave background radiation, and close to the local energy density in starlight (Wdowczyk and Wolfendale, 1989; Longair, 2002).

We are witnessing an era of strong development of new telescopes with higher and higher resolution allowing new observing techniques so as to constrain the CR flux at energies lower than about $1 \mathrm{GeV}$. Detections of $\mathrm{OH}^{+}$and $\mathrm{H}_{2} \mathrm{O}^{+}$ in low $\mathrm{H}_{2}$ fraction regions (Neufeld et al., 2010; Gerin et al.,
2010), enhanced CR ionisation rate (hereafter $\zeta^{\mathrm{H}_{2}}$ ) in molecular clouds close to supernova remnants (Becker et al., 2011; Ceccarelli et al., 2011), observations of $\mathrm{H}_{3}^{+}$in diffuse clouds (Indriolo and McCall, 2012) and towards the Galactic centre (Goto et al., 2008) as well as $\gamma$ luminosity of molecular clouds (Montmerle, 2010) pose the question about how to reconcile the high values of $\zeta^{\mathrm{H}_{2}}$ estimated in diffuse regions $\left(\zeta^{\mathrm{H}_{2}} \sim 10^{-15}-10^{-16} \mathrm{~s}^{-1}\right)$ with those ones measured in denser clouds $\left(\zeta^{\mathrm{H}_{2}} \sim 10^{-17}-10^{-18} \mathrm{~s}^{-1}\right)$.

\section{The role of cosmic rays on physics and chemical composition of molecular clouds}

The study of the interaction of CRs with the ISM is a glaring example of a multidisciplinary task involving the analysis of several physical and chemical processes. In a prestellar core, the primary source of ionisation is represented by CRs, since X-rays ionisation arises only in presence of embedded young stellar objects (Krolik and Kallman, 1983; Silk and Norman, 1983) and interstellar UV photons are absorbed in a thin layer of about 4 magnitudes of visual extinction (McKee, 1989). The key quantity that governs the interstellar chemistry, namely the creation of more and more complex 


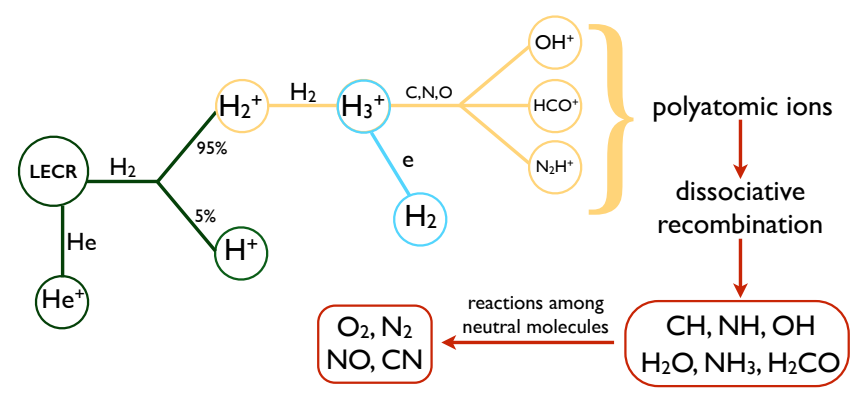

Figure 1. The ionisation of molecular hydrogen due to an interaction with a low-energy CR (LECR) leads to the formation of more and more complex molecules that we can observe in molecular clouds. In particular, in diffuse clouds, where the visual extinction $A_{\mathrm{V}}$ is about $1 \mathrm{mag}$, the main reaction channel yields the trihydrogen cation $\left(\mathrm{H}_{3}^{+}\right)$that rapidly recombines with electrons. In denser regions $\left(A_{\mathrm{V}}>3-4 \mathrm{mag}\right) \mathrm{H}_{3}^{+}$reacts with heavier elements creating polyatomic ions up to neutral molecules, among which ammonia and water.

molecules in molecular clouds, is the so-called cosmic-ray ionisation rate, that is the number of hydrogen molecule ionisation per second (see e.g. Wakelam et al., 2010). CRs interact with dense molecular clouds by ionising their main component, the molecular hydrogen, and this process activates the chemistry observed in clouds. Since the ionisation cross section of $\mathrm{H}_{2}$ by collisions with electrons and protons has a maximum at $\sim 50 \mathrm{eV}$ and $\sim 10 \mathrm{keV}$ (Padovani et al., 2009), respectively, the bulk of ionisation is due to low-energy CRs, namely particles with energy lower than about $100 \mathrm{MeV}-$ $1 \mathrm{GeV}$ (see Fig. 1).

In turn, the ionisation fraction, that is the quantity of charged particles with respect to neutrals that is proportional to $\sqrt{\zeta^{\mathrm{H}_{2}}}$ (McKee and Ostriker, 2007), controls the coupling of magnetic fields with the gas, driving the dissipation of turbulence and angular momentum transfer, thus playing a crucial role in protostellar collapse and the dynamics of accretion discs (e.g. Balbus and Hawley, 1991; Padovani et al., 2013).

CRs also represent an important source of heating for molecular clouds. In fact, inelastic collisions with interstellar molecules and atoms convert about half of the energy of primary and secondary electrons yielded by the ionisation process into heat (e.g. Glassgold and Langer, 1973; Glassgold et al., 2012).

During the last 50 years, several values of $\zeta^{\mathrm{H}_{2}}$ ranging from a few $10^{-16} \mathrm{~s}^{-1}$ to a few $10^{-18} \mathrm{~s}^{-1}$ have been observationally determined in diffuse and dense interstellar clouds from measurements of the abundances of various chemical species (see Fig. 6 in Padovani and Galli, 2013, and references therein). Nevertheless, the lower limit of $\zeta^{\mathrm{H}_{2}} \sim$ $10^{-17} \mathrm{~s}^{-1}$ computed by Spitzer Jr. and Tomasko (1968) is commonly used in chemical and magnetohydrodynamic (hereafter MHD) as the "standard" $\zeta^{\mathrm{H}_{2}}$ in molecular clouds.
Determining $\zeta^{\mathrm{H}_{2}}$ from Earth is complicated because the interplanetary magnetic field and the solar wind prevent lowenergy CRs from entering the heliosphere (solar modulation). This means that Earth-based measurements of CR fluxes provide only a lower limit on the interstellar spectrum of protons and heavy nuclei for energies below $1 \mathrm{GeV} /$ nucleon. Besides, the low-energy CR electron flux, which already fluctuates in the energy range of $10-100 \mathrm{GeV}$ (see e.g. Casadei and Bindi, 2004), is damped by solar modulation. This means that it is extremely difficult to know what happens to the $\mathrm{CR}$ spectrum below $\mathrm{GeV}$ energies, representing the main constraint for any trustworthy estimate of the CR ionisation rate in the ISM. Only when the spacecrafts Voyager 1 and 2 will be far beyond the heliopause, the outermost boundary for solar modulation effects lying at $130-150 \mathrm{AU}$ from the Sun, it will be possible to measure the low-energy CR spectrum. On 25 August 2012, Voyager 1 reached the heliopause and now we have information about the CR interstellar spectrum down to energies of about $1 \mathrm{MeV}$ (Potgieter et al., 2013) and $10 \mathrm{MeV}$ (Potgieter, 2013) for Galactic electrons and protons, respectively, but still not enough to constrain the low-energy cosmic-ray flux. Since Voyager 1 is escaping the solar system at a speed of about 3.6 AU per year, in about 5 years from now we will be able to look at the true interstellar spectrum.

\section{Energy losses and magnetic effects on cosmic-ray propagation}

While crossing a molecular cloud, CRs undergo collisions with $\mathrm{H}_{2}$ molecules. According to their initial energy and their composition, they are slowed down due to processes that are specific of a particular kind of particle (bremsstrahlung, synchrotron emission, and inverse Compton scattering for electrons; elastic interactions, pion production, and spallation for protons) or common both to CR protons and electrons (Coulomb and inelastic interactions, and ionisation). The energy loss function for the species $k$ is defined as

$L_{k}\left(E_{k}\right)=-\frac{1}{n\left(\mathrm{H}_{2}\right)}\left(\frac{\mathrm{d} E_{k}}{\mathrm{~d} \ell}\right)$,

where $n\left(\mathrm{H}_{2}\right)$ is the density of the medium in which the particle of energy $E_{k}$ propagates and $\ell$ is the path length. Figure 2 shows the energy loss functions for protons and electrons colliding with molecular hydrogen.

While in the past it was assumed a lower cutoff for CR energy to compute $\zeta^{\mathrm{H}_{2}}$ (e.g. Nath and Biermann, 1994), in Padovani et al. (2009) we showed that even if a local interstellar spectrum is lacking of low-energy particles, the slowing-down of CR protons and electrons during their propagation produces a low-energy tail. Our modelling is able to explain the decrease of $\zeta^{\mathrm{H}_{2}}$ with increasing hydrogen column density computed from observations. In particular, a proton component at low energies, and most likely also an electron component, could be necessary to reproduce the data. 


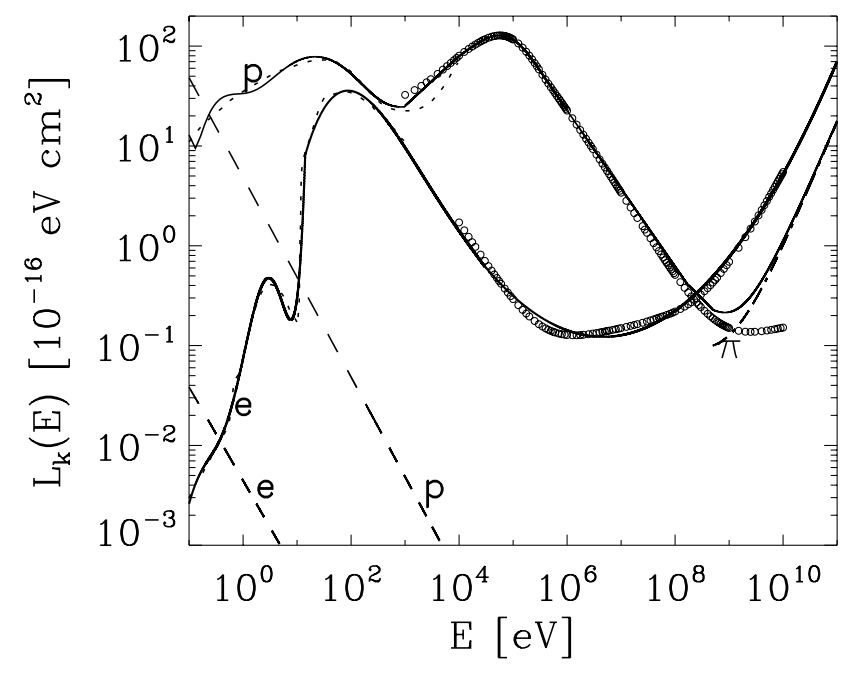

Figure 2. Energy loss functions $L_{\mathrm{e}}\left(E_{\mathrm{e}}\right)$ and $L_{\mathrm{p}}\left(E_{\mathrm{p}}\right)$ for electrons and protons, respectively, colliding with $\mathrm{H}_{2}$ (solid curves), compared with NIST data (circles); dashed curves show Coulomb losses for a fractional electron abundance $n_{\mathrm{e}} / n\left(\mathrm{H}_{2}\right)=10^{-7}$; dash-dotted curve labelled with $\pi$ represents the energy loss by pion production computed following Schlickeiser (2002); dotted curves show the results by Phelps (1990) and Dalgarno et al. (1999) for $p-\mathrm{H}_{2}$ and $e-\mathrm{H}_{2}$, respectively.

In Padovani and Galli (2011), we studied how the presence of magnetic fields affects the propagation of CRs. In fact, being charged particles, CRs moves along field lines following an helicoidal path. This means that they "see" a larger column density of molecular hydrogen with respect to a rectilinear propagation, given by

$N(\alpha)=\int_{0}^{\ell_{\max }(\alpha)} n(\ell) \mathrm{d} \ell$,

where $\ell_{\max }$ is the maximum depth reached inside the core and $n(\ell)$ is the $\mathrm{H}_{2}$ volume density. The angle $\alpha$, called pitch angle, is the angle between the $\mathrm{CR}$ velocity and the direction of the magnetic field and its evolution during the CR propagation reads

$\alpha=\arccos \sqrt{1-\chi+\chi \cos ^{2} \alpha_{\mathrm{ICM}}}$,

where $\chi=B / B_{\mathrm{ICM}}$ is the ratio between the local and the intercloud magnetic field. The two competing effects arising from the presence of magnetic fields are magnetic focusing that increases the CR flux where the field is more concentrated, and magnetic mirroring according to which CRs are bounced out of the cloud when the pitch angle reaches $\pi / 2$, namely when the CR velocity is perpendicular to the field line.

\section{Cosmic rays in collapsing clouds}

The decrease of $\zeta^{\mathrm{H}_{2}}$ in the densest central regions of a protostellar core may have a strong impact on the decoupling between gas and magnetic field, leading the core towards the collapse. Mellon and Li (2009) propose that the attenuation of $\zeta^{\mathrm{H}_{2}}$ down to $10^{-18} \mathrm{~s}^{-1}$ may increase the ambipolar diffusion having consequences on the formation of a rotationally supported disc.

Following our previous studies (Padovani et al., 2009; Padovani and Galli, 2011) where we accurately examined the CR propagation accounting for column density and magnetic effects, in Padovani et al. (2013) we investigated the propagation of CRs in the inner 300-400 AU of a cloud core where the formation of a protostellar disc is expected. In particular, we considered density and magnetic field configurations obtained by ideal-MHD numerical simulations related to a rotating collapsing core (Joos et al., 2012), performed with the AMR code RAMSES (Teyssier, 2002; Fromang et al., 2006). One could deduce that magnetic effects are negligible when CRs reach the inner part of a core (inside a radius of $\sim 500 \mathrm{AU}$ ). In fact they may already be in the regime of exponential attenuation $\left(N>10^{25} \mathrm{~cm}^{-2}\right)$ since they have passed through a large amount of column density (see Fig. 1 in Padovani et al., 2013). On the contrary, we found that even at very high densities magnetic fields can efficiently remove CRs.

It is not possible to quantify to what extent column density effects dominate over magnetic effects since it depends on the field configuration considered. However, we did an estimate by calculating $\zeta^{\mathrm{H}_{2}}$ both accounting and neglecting magnetic effects. Figure 4 shows how magnetic shielding determines a decrease of $\zeta^{\mathrm{H}_{2}}$ by a factor of $\sim 10$ at a radius of 300-400 AU and how the central region, where the minimum $\zeta^{\mathrm{H}_{2}}$ is reached, increases in size from $\sim 10$ to $\sim 50 \mathrm{AU}$. This example also demonstrates that the use of the constant "standard" value $\zeta^{\mathrm{H}_{2}}=10^{-17} \mathrm{~s}^{-1}$ overestimates the CR ionisation rate in the densest region of a molecular cloud. Running our code for different initial conditions (see Table 1 in Padovani et al., 2013), we found a decrease of $\zeta^{\mathrm{H}_{2}}$ below $10^{-18} \mathrm{~s}^{-1}$ in the central 300-400 AU, where $n \gtrsim 10^{9} \mathrm{~cm}^{-3}$, if the toroidal component is larger than about $40 \%$ of the total field and in the cases of low and intermediate ionisation (mass-to-flux ratio ${ }^{1} \lambda=17$ and 5, respectively).

In order to avoid running the whole code, we also formulated a general fitting expression to approximately compute $\zeta^{\mathrm{H}_{2}}$ as a function of the column density, toroidal-to-poloidal magnetic field ratio, and magnetic field strength (see Sect. 6 in Padovani et al., 2013).

\footnotetext{
${ }^{1}$ It is a non-dimensional value that gives information on the level of magnetisation.
} 


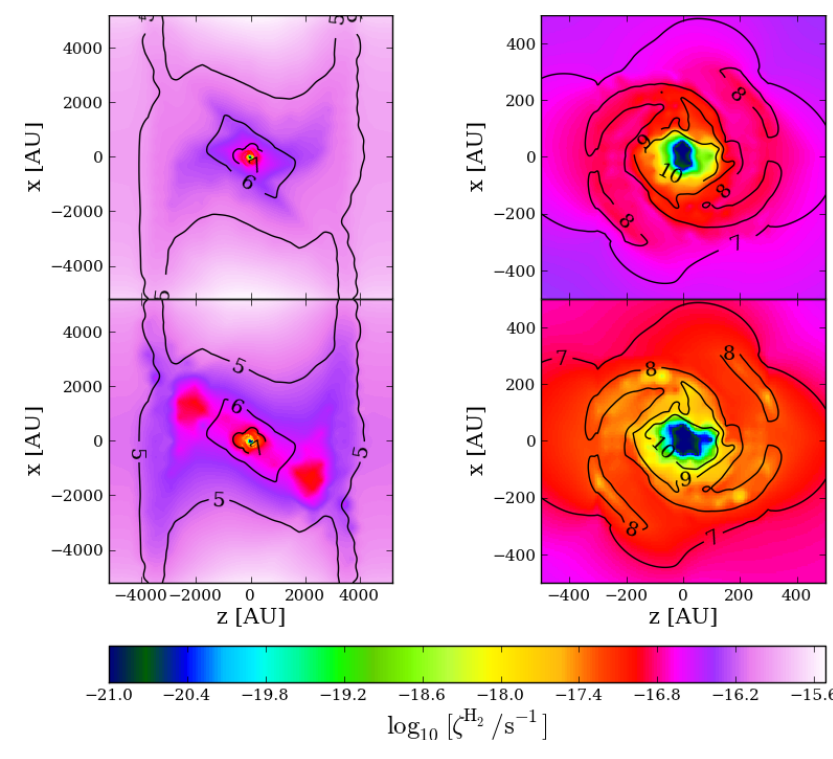

Figure 3. CR ionisation maps and iso-density contours (black solid lines) for the non-magnetic (upper row) and magnetic (lower row) cases. This model refers to a perpendicular rotator, namely the main direction of the magnetic field and the rotation axis are perpendicular, with a mass-to-flux ratio $\lambda=5$ (see Fig. 10 in Padovani et al., 2013, for more details). Left panels show the entire computational domain while right panels show a zoom in the inner region. Labels show $\log _{10}\left[n / \mathrm{cm}^{-3}\right]$, where $n$ is the number density of $\mathrm{H}_{2}$.

\section{Conclusions}

Cosmic rays constitute the main ionising and heating agent in dense, starless, molecular cloud cores. We reexamined the physical quantities necessary to determine the CR ionisation rate (especially the $\mathrm{CR}$ spectrum below $1 \mathrm{GeV}$ and the ionisation cross sections), and calculated $\zeta^{\mathrm{H}_{2}}$ as a function of the $\mathrm{H}_{2}$ column density. We also accounted for magnetic effects, finding that mirroring and focusing define the spatial domain where CRs can determine the coupling between gas and magnetic fields.

Even if we are aware that the CR propagation should be computed simultaneously with the MHD simulation, our study represents an important proof of concept. In fact, we showed that the inclusion of magnetic effects is essential to account for the true path of CRs during their propagation. We found that, in the densest region of a protostellar core, $\zeta^{\mathrm{H}_{2}}$ can be reduced of about $10^{3}$ times the "standard" value of $10^{-17} \mathrm{~s}^{-1}$, down to the lower limit set by shortlived radionuclides in protoplanetary discs (Umebayashi and Nakano, 1981; Cleeves et al., 2013).

When the dynamical evolution becomes slower than the diffusion of the magnetic field, the magnetic braking becomes inefficient. This is predicted for densities larger that $10^{12} \mathrm{~cm}^{-3}$ by non-ideal MHD models (Dapp et al., 2012). We noticed that in our models the decrease of $\zeta^{\mathrm{H}_{2}}$ can occur in some cases even at lower densities $\left(n>10^{9} \mathrm{~cm}^{-3}\right)$, resulting in very low ionisation fractions. The consequences of the reduced $\mathrm{CR}$ ionisation rate on the magnetic diffusion coefficients are analysed in detail in Padovani et al. (2014).

Acknowledgements. M. Padovani thanks K. Scherer, H. Fichtner, D. Bomans, K. Weis, and J. Tjus for the invitation to this workshop. M. Padovani and P. Hennebelle acknowledge the financial support of the Agence National pour la Recherche (ANR) through the COSMIS project.

Edited by: J. Tjus

Reviewed by: two anonymous referees

\section{References}

Balbus, S. A. and Hawley, J. F.: A powerful local shear instability in weakly magnetized disks. I - Linear analysis. II - Nonlinear evolution, Astrophys. J., 376, 214-222, 1991.

Becker, J. K., Black, J. H., Safarzadeh, M., and Schuppan, F.: Tracing the Sources of Cosmic Rays with Molecular Ions, Astrophys. J., 739, L43, doi:10.1088/2041-8205/739/2/L43, 2011.

Casadei, D. and Bindi, V.: The Origin of Cosmic Ray Electrons and Positrons, Astrophys. J., 612, 262-267, 2004.

Ceccarelli, C., Hily-Blant, P., Montmerle, T., Dubus, G., Gallant, Y., and Fiasson, A.: Supernova-enhanced Cosmic-Ray Ionization and Induced Chemistry in a Molecular Cloud of W51C, Astrophys. J. Lett., 740, L4, doi:10.1088/2041-8205/740/1/L4, 2011.

Cleeves, L. I., Adams, F. C., Bergin, E. A., and Visser, R.: Radionuclide Ionization in Protoplanetary Disks: Calculations of Decay Product Radiative Transfer, Astrophys. J., 777, 28, doi:10.1088/0004-637X/777/1/28, 2013.

Dalgarno, A., Yan, M., and Liu, W.: Electron Energy Deposition in a Gas Mixture of Atomic and Molecular Hydrogen and Helium, Astrophys. J. Suppl. S., 125, 237-256, 1999.

Dapp, W. B., Basu, S., and Kunz, M. W.: Bridging the gap: disk formation in the Class 0 phase with ambipolar diffusion and Ohmic dissipation, Astron. Astrophys., 541, A35, doi:10.1051/00046361/201117876, 2012.

Fromang, S., Hennebelle, P., and Teyssier, R.: A high order Godunov scheme with constrained transport and adaptive mesh refinement for astrophysical magnetohydrodynamics, Astron. Astrophys., 457, 371-384, doi:10.1051/0004-6361:20065371, 2006.

Gerin, M., De Luca, M., Black, J., et al.: Interstellar $\mathrm{OH}^{+}, \mathrm{H}_{2} \mathrm{O}^{+}$ and $\mathrm{H}_{3} \mathrm{O}^{+}$along the sight-line to G10.6-0.4, Astron. Astrophys., 518, L110, doi:10.1051/0004-6361/201014576, 2010.

Glassgold, A. E. and Langer, W. D.: Heating of MolecularHydrogen Clouds by Cosmic Rays and X-Rays, Astrophys. J., 186, 859-888, 1973.

Glassgold, A. E., Galli, D., and Padovani, M.: Cosmic-Ray and XRay Heating of Interstellar Clouds and Protoplanetary Disks, Astrophys. J., 756, 157, doi:10.1088/0004-637X/756/2/157, 2012.

Goto, M., Usuda, T., Nagata, T., Geballe, T. R., McCall, B. J., C. P.,Indriolo, N., Suto, H., Henning, T., Morong, C. P., and Oka, T.: Absorption Line Survey of $\mathrm{H}_{3}^{+}$toward the Galactic Center Sources. II. Eight Infrared Sources within $30 \mathrm{pc}$ of the Galactic Center 2008, Astrophys. J., 688, 306, doi:10.1086/591657, 2008. 
Indriolo, N. and McCall, B. J.: Investigating the Cosmic-Ray Ionization Rate in the Galactic Diffuse Interstellar Medium Through Observations of $\mathrm{H}_{3}^{+}$, Astrophys. J., 745, 91, doi:10.1088/0004637X/745/1/91, 2012.

Joos, M., Hennebelle, P., and Ciardi, A.: Protostellar disk formation and transport of angular momentum during magnetized core collapse, Astron. Astrophys., 543, A128, doi:10.1051/00046361/201118730, 2012.

Krolik, J. H. and Kallman, T. R.: X-ray ionization and the Orion molecular cloud, Astrophys. J., 267, 610-624, 1983.

Longair, M. S.: High Energy Astrophysics, Cambridge University Press, Cambridge, UK, 2002.

McKee, C. F.: Photoionization-regulated star formation and the structure of molecular clouds, Astrophys. J., 345, 782-801, doi:10.1086/167950, 1989.

McKee, C. F. and Ostriker, E. C.: Theory of Star Formation, Annu. Rev. Astron. Astr., 45, 565-687, doi:10.1146/annurev.astro.45.051806.110602, 2007.

Mellon, R. R. and Li, Z.-H.: Magnetic Braking and Protostellar Disk Formation: Ambipolar Diffusion, Astrophys. J., 698, 922, doi:10.1088/0004-637X/698/1/922 2009.

Montmerle, T.: Gamma-rays from star-forming regions: from SNOBs to Dark Accelerators, in: High Energy Phenomena in Massive Stars, San Francisco, CA, edited by: Martí, J., LuqueEscamilla, P. L., and Combi, J. A., ASP Conf. Ser. 422, p. 85, 2010.

Nath, B. B. and Biermann, P. L.: Cosmic ray ionization of the interstellar medium, Mon. Not. R. Astron. Soc., 267, 447-451, 1994.

Neufeld, D. A., Goicoechea, J. R., Sonnentrucker, P., et al.: Herschel/HIFI observations of interstellar $\mathrm{OH}^{+}$and $\mathrm{H}_{2} \mathrm{O}^{+}$towards W49N: a probe of diffuse clouds with a small molecular fraction, Astron. Astrophys., 521, L10, doi:10.1051/00046361/201015077, 2010

Padovani, M. and Galli, D.: Effects of magnetic fields on the cosmic-ray ionization of molecular cloud cores, Astron. Astrophys., 530, A109, doi:10.1051/0004-6361/201116853, 2011.

Padovani, M. and Galli, D.: Cosmic-Ray Propagation in Molecular Clouds, in: Cosmic Rays in Star-Forming Environments, edited by: Torres, D. F. and Reimer, O., Advances in Solid State Physics, Springer, Berlin, 34, 61-82, 2013.
Padovani, M., Galli, D., and Glassgold, A. E.: Cosmic-ray ionization of molecular clouds, Astron. Astrophys., 501, 619-631, 2009.

Padovani, M., Hennebelle, P., and Galli, D.: Cosmic-ray ionisation in collapsing clouds, Astron. Astrophys., 560, A114, doi:10.1051/0004-6361/201322407, 2013.

Padovani, M., Galli, D., Hennebelle, P., Commerçon, B., and Joos, M.: The role of cosmic rays on magnetic field diffusion and the formation of protostellar discs, Astron. Astrophys., submitted, 2014.

Phelps, A. V.: Cross Sections and Swarm Coefficients for $\mathrm{H}^{+}, \mathrm{H}_{2}^{+}$, $\mathrm{H}_{3}^{+}, \mathrm{H}, \mathrm{H}_{2}$, and $\mathrm{H}^{-}$in $\mathrm{H}_{2}$ for Energies from $0.1 \mathrm{eV}$ to $10 \mathrm{keV}$, J. Phys. Chem. Ref. Data, 19, 653, doi:10.1063/1.555858, 1990.

Potgieter, M. S.: Very local interstellar spectra for galactic electrons, protons and helium, in: 33rd ICRC, Rio de Janeiro, Brazil, 2013, The Astroparticle Physics Conference, arXiv:1310.6133v1, 2013.

Potgieter, M. S., Vos, E. E., Nndanganeni, R. R., Boezio, M., and Munini, R.: A Very Local Interstellar Spectrum for Galactic Electrons, in: 33rd ICRC, Rio de Janeiro, Brazil, 2013, The Astroparticle Physics Conference, arXiv:1308.1591v1, 2013.

Schlickeiser, R.: Cosmic Ray Astrophysics, Springer Berlin, 2002.

Silk, J. and Norman, C.: X-ray emission from pre-main-sequence stars, molecular clouds and star formation, Astrophys. J. Lett., 272, L49-L53, 1983.

Spitzer Jr., L. J. and Tomasko, M. G.: Heating of H i Regions by Energetic Particles, Astrophys. J., 152, p. 971, doi:10.1086/149610, 1968.

Teyssier, R.: Cosmological hydrodynamics with adaptive mesh refinement, Astron. Astrophys., 385, 337-364, doi:10.1051/00046361:20011817, 2002.

Umebayashi, T. and Nakano, T.: Fluxes of Energetic Particles and the Ionization Rate in Very Dense Interstellar Clouds, Publ. Astron. Soc. Jpn., 33, 617-635, 1981.

Wakelam, V., Herbst, E., Le Bourlot, J., Hersant, F., Selsis, F., and Guilloteau, S.: Sensitivity analyses of dense cloud chemical models, Astron. Astrophys., 517, A21, doi:10.1051/00046361/200913856, 2010.

Wdowczyk, J. and Wolfendale, A. W.: Highest energy cosmic rays, Annu. Rev. Nucl. Part. S., 39, 43-71, doi:10.1146/annurev.ns.39.120189.000355, 1989. 